\title{
Fatigue analysis of riveted railway bridge connections using the theory of critical distances
}

\author{
Timothy D. Righiniotis, Boulent M. Imam*and Marios K. Chryssanthopoulos \\ Faculty of Engineering and Physical Sciences, University of Surrey, Guildford, GU2 \\ $7 X H$, United Kingdom \\ ${ }^{*}$ Corresponding Author. Tel: +44-1483-686652; Fax: +44-1483-450984: Email address: \\ b.imam@surrey.ac.uk; Postal address: Faculty of Engineering and Physical Sciences (C5), University \\ of Surrey, Guildford, Surrey, GU2 7XH, United Kingdom
}

\begin{abstract}
Recent studies have found that stringer-to-cross-girder connections in riveted railway bridges are susceptible to fatigue cracking, caused by secondary, deformation induced effects. These effects are difficult to interpret in terms of a single applied stress descriptor, which is customarily used in an S-N assessment. In order to address this problem, the results of a global-local finite element analysis of a riveted railway bridge are used in this paper within the context of the theory of critical distances (TCD). Using the TCD in the way proposed by Taylor [1], fatigue damage (a) is shown to converge upon mesh refinement and (b) is found to be relatively sensitive to the selection of the characteristic dimension of the critical volume. Furthermore, comparisons of the TCD-based method with its more traditional, detail-specific S-N counterpart, reveal that the latter can underestimate fatigue damage, in some cases by a factor of 3.5 .
\end{abstract}

Keywords: Bridges; fatigue; finite element method; stress concentrations; Theory of Critical Distances.

\section{Introduction}

Fatigue of riveted railway bridges has received considerable attention during the past decade or so, not least because of their age ( $>100$ years) and their large number both in Europe and North America. The majority of reported fatigue damage cases have been linked to stringer-to-cross-girder connections [2,3]. Fatigue damage in these connections has been attributed to secondary, deformation induced effects, which were certainly not considered in the design of the bridges. Even today, these 
effects are very difficult to account for and often overlooked in a standard, code-based S-N treatment $[4,5]$.

Despite the substantial number of fatigue tests on riveted details, very few of these tests have been conducted on stringer-to-cross-girder connections. Due to the complex behaviour of these connections, which also depends on the interaction of different components such as rivets, angle clips and parts of the primary bridge members such as flanges and webs, it is difficult to define a characteristic ('nominal') stress value and hence express the tests results in a meaningful way on an S$\mathrm{N}$ basis.

With the advent of sophisticated finite element (FE) analysis, the focus, in a number of bridgerelated applications, has shifted to the examination of local stresses in the vicinity of stress concentrations. For example, the present authors, using sub-modelling techniques and threedimensional (3D) bricks, considered the principal stress histories within a typical bridge connection [6]. These principal stress histories coupled with a generic S-N curve led to the calculation of the fatigue life.

However, as pointed out in [6] and elsewhere [7], a major drawback of the FE method is that, in general, the maximum stress at the points of stress concentration does not converge with increasing mesh density since these points are usually points associated with numerical singularities. An alternative to overcome this problem is to carry out an elastic-plastic analysis [1,8] but this is computationally very demanding. Another approach, which has been developed recently for welded details, is the so-called 'structural stress' method in which an equivalent stress state is defined at some distance from the stress concentration and considered for fatigue calculations [7]. The 'hot spot stress' method, which has been developed for offshore applications and involves extrapolation of stresses, is, yet another alternative [9]. However, the last two methods have been found to have certain limitations for 3D stress concentrations [10] and it is far from straightforward to define the equivalent stress state for several cases, including non-welded details.

During recent years, the Theory of Critical Distances (TCD), which considers the elastic stress distribution over a certain 'critical distance' ahead of the stress concentration, has been developed for the fatigue analysis of notched components $[8,11]$. This theory, which can be used in conjunction with linear elastic FE analyses and be applied to any type of stress concentration, has the advantage of requiring only a limited number of fatigue physical test data. 
In this paper, the TCD is applied to various parts of the bridge connection investigated previously [6,12]. For this reason, the BS 5400 medium traffic trains [4] are initially traversed over a short span bridge. Following the FE analysis, histories of the average, over a volume, principal stress are obtained at various critical locations within the connection and the fatigue damage is estimated using the plain (un-notched) material S-N curve and Miner's rule. The method is tested in terms of its convergence characteristics as well as its sensitivity to the selection of the size of the critical volume. Having explored the method's robustness, fatigue life estimates are obtained using the TCD and compared to the estimates obtained from the more traditional S-N approach, which relies on the nominal stress.

\section{The theory of critical distances (TCD)}

The concept behind the TCD is that the fatigue behaviour of notches does not depend on a single stress concentration. Rather, fatigue is influenced by the stress distribution ahead of the stress concentration and, therefore, on the stress gradient $[1,8,11]$. The idea was initially put forward during the middle of the previous century by Neuber [13] and Peterson [14]. Neuber proposed the use of a reference stress averaged along a line of a given length (line method). Peterson simplified this further by considering the stress at a point, which is located a certain distance ahead of the stress concentration (point method). With the extensive development and use of the FE method during recent years, which allows the analysis of complex, 3D geometries, reasonably accurate descriptions of the stress fields ahead of stress concentrations can be obtained. This fact, combined with the advances in the field of Linear Elastic Fracture Mechanics (LEFM), has led, during the last decade, to the development of other critical distance methods $[8,11]$.

A key feature in any such method is the definition of the critical distance. This distance is characteristic of the zone within which the physical processes leading to fatigue crack initiation are presumed to take place. This suggests that, unlike the traditional theory, which relies on stress definition at a single point, damage within the TCD context is associated with a finite volume. Moreover, by linking the fatigue limit $\Delta \sigma_{0}$ of an un-notched specimen to the threshold stress intensity $\Delta K_{t h}$, the critical distance $L$ may be calculated according to [11]

$$
L=\frac{1}{\pi}\left(\frac{\Delta K_{t h}}{\Delta \sigma_{0}}\right)^{2}
$$


It has to be noted that, since $\Delta K_{t h}$ and $\Delta \sigma_{0}$ are both material properties, Eq. (1) implies that $L$ will also be a material property.

Having established $L$, the next step is to define an appropriate stress measure (characteristic stress), which is associated with $L$ and used in fatigue calculations. Different methods have been proposed in the past to that effect. A method which is akin to Peterson's approach considers the maximum principal stress $\sigma_{p(\max )}$ at a single point (point method), at a distance of $L / 2$ from the tip of the notch, as shown in Fig. 1(a) [1,11]. A somewhat different method is the line method, which averages the maximum principal stresses $\sigma_{p(\max )}$ along a line of length $2 L$, as shown in Fig. 1(b). The averaging of principal stresses is carried out according to [1,11]

$$
\sigma_{\text {ave }}=\frac{1}{2 L} \int_{0}^{2 L} \sigma_{p(\max )}(r) d r
$$

A third method is the semi-circular area (area method), which averages principal stresses over a semicircular region. This region is centred at the notch tip and has a radius of $1.32 L$ [1].

While the previously mentioned methods are suitable for tackling 2D geometries, for 3D applications, Taylor and his co-workers [1] have proposed the use of the volume method, which relies on averaging principal stresses over a hemisphere centred at the tip of the notch with a radius of $1.54 \mathrm{~L}$. The constants 1.32 and 1.54, associated with the area and volume methods, respectively, arise from the area and volume integration of the crack tip singular stress fields [1].

Once the characteristic stress is obtained, it is then compared to the Constant Amplitude Fatigue Limit (CAFL) of the plain (un-notched) material in order to determine whether fatigue failure will occur.

By comparing analytical and experimental results, the accuracy of the point, line and area methods in predicting the CAFL of notched components has been tested extensively by Taylor and other researchers [11,15-18]. These investigations were carried out on a wide range of notch geometries such as circular holes [11,15], corner fillets [16,17], semi-circular edge notches and centre notches ranging from blunt to sharp [15,18]. The validity of the TCD has been tested for different materials, such as steel $[11,15,16,18]$, cast iron $[15,17,18]$ and aluminium $[15,18]$. Furthermore, the TCD has been applied to a number of components ranging from cylindrical bars $[15,18]$ and plates $[11,15,18]$ to car suspension arms [16] and ship components [17]. These investigations have 
demonstrated that the difference between the CAFL predicted by the TCD compared with the experimental values is within $20 \%$.

While the previously mentioned investigations were carried out for constant amplitude loading, it was shown in [16] that the TCD is also capable of predicting fatigue life under variable amplitude loading by using the characteristic stress in conjunction with the plain material S-N curve. The accuracy of the TCD for the fatigue assessment of welded joints [19,20], under multiaxial stress conditions [21,22] and for fretting fatigue problems [23] has also been verified.

Following this brief discussion on the TCD, the FE analysis is presented in the next section. In the present investigation, the TCD is used in conjunction with variable amplitude loading resulting from the passage of trains over a short-span, riveted bridge in order to estimate the fatigue damage and the remaining life of a critical connection.

\section{Finite element analysis}

\subsection{Bridge finite element model}

The global-local FE model of the bridge is shown in Fig. 2(a). Fig. 2(b) depicts in detail the connection shown in Fig. 2(a) as well as the nomenclature used to identify its components. The entire model, which was described in more detail and validated in [6], consists of approximately 67000 brick elements and 20000 shell elements. The FE analyses are carried out assuming linear elastic material behaviour. Two levels of clamping are assumed for all rivets, i.e. a low to moderate clamping stress of $100 \mathrm{MPa}$ and a high clamping stress of $200 \mathrm{MPa}$.

The bridge is loaded with the BS 5400 medium traffic trains [4], which are traversed in $1 \mathrm{~m}$ steps over one track of the bridge, as shown in Fig. 2(a), up to the point of load repetition caused by the passage of similar wagons following the engine car. The number of analysis steps vary between 35 and 45 depending on the train traversed over the bridge. The train axle loads are applied quasi-statically directly onthe top flange of the stringers thus neglecting dynamic amplification and any beneficial effects associated with the load spread on the rails and sleepers.

\subsection{Material properties}

The bridge is assumed to be made from wrought iron, a material that was extensively used in the mid- to latter part of the $19^{\text {th }}$ century. The value of Young’s modulus for wrought iron has been found 
to vary between about 170 and $220 \mathrm{GPa}$ [24-26], having an average value of approximately $200 \mathrm{GPa}$.

This value is used here together with a Poisson's ratio of 0.3.

With regard to the threshold stress intensity range, a value of $\Delta K_{t h}=13.5 \mathrm{MPa} \cdot \mathrm{m}^{1 / 2}$ is assumed. This value was suggested in [27] following a series of crack growth experiments on wrought iron at a load ratio of 0.1 . For the fatigue limit $\Delta \sigma_{0}$ of plain wrought iron material, the value of $183 \mathrm{MPa}$ is used, which is based on fatigue tests at a load ratio of 0.1 [24]. There are only limited experimental data on fatigue properties of this material, and the values proposed herein should be used with caution.

By using the assumed values of $\Delta K_{t h}$ and $\Delta \sigma_{0}$ in Eq. (1), a critical distance value of $L=1.73 \mathrm{~mm}$ is obtained.

\subsection{Fatigue damage calculation}

In a previous investigation [6], the critical regions within the connection were found to be typically located along the perimeter of the rivet holes on the face of the connection angle in contact with the cross-girder and stringer webs, along the angle fillet, and around the perimeter of the rivets at the head-to-shank intersection.

Fig. 3 shows a close-up view of a fatigue-critical region on the perimeter of one of the angle holes. Dotted lines indicate the boundary of the critical volume, whose characteristic dimension is 1.54L. Since part of the critical region at the hole perimeter is formed by a right angle corner, a quarter of a sphere is used in this case as the critical volume (see Fig. 3). Within the critical space, those brick elements that lie on the boundary are considered in further calculations when at least half of their volume lies within the critical volume (see shaded areas in Fig. 3). The same assumption is also applied to the other parts of the connection such as the angle fillet (see Fig. 4) and rivet (see Fig. 5). Again, in both Fig. 4 and 5 the shaded regions depict the elements used for stress calculation. Within these elements, the maximum principal stresses are obtained at their centroids since these represent an average value over the element. The stress values of interest are then calculated by averaging, over all the elements, the centroidal stresses.

It has to be mentioned that notch tips, and hence their respective volumes, are, in general, not unique. For example, in Fig. 3 notch tips are located all around the circumference at the surface of the hole. In the same manner, several notch tips, and hence several critical volumes, may be identified along the depth of the angle fillet in Fig. 4 and around the circumference of the rivet head-to-shank 
intersection (Fig. 5). In order to obtain the stress history, which is used in subsequent fatigue damage calculations, the procedure is applied at each load step of the FE analysis. Several stress histories are thus generated corresponding to the different notch tips. Fig. 6(a) depicts a typical stress ( $\left.\sigma_{\text {ave }}\right)$ history at the edge of a hole. For each of these stress histories, the rainflow counting method is used to convert them into stress range $\left(\Delta \sigma_{\text {ave }}\right)$ blocks and Miner's rule is applied on the plain material S-N curve in order to calculate the fatigue damage.

Considering the very limited number of experimental data on plain wrought-iron material near the fatigue limit, a single-slope S-N curve (slope $1 / 5$ ) with a fatigue limit of $\Delta \sigma_{0}=183 \mathrm{MPa}$, defined at $N_{F L}=2 \times 10^{6}$ [24], is used for damage calculations (see Fig. 6(b)). Since train loading is of variable amplitude loading (see Fig. 6(a)), the S-N curve is extended below $\Delta \sigma_{0}$. Thus, the fatigue damage for a single train passage, which produces $k$ stress blocks, will be given as

$$
D_{l 1}=\frac{1}{N_{F L}\left(\Delta \sigma_{0}\right)^{5}} \sum_{i=1}^{k} n_{i}\left[\left(\Delta \sigma_{\text {ave }}\right)_{i}\right]^{5}
$$

where $\left(\Delta \sigma_{\text {ave }}\right)_{i}$ is the $i^{\text {th }}$ stress range and $n_{i}$ is the corresponding number of cycles. It is worth mentioning that, on the basis of the calculated stress histories, the load ratio $R$ was found to vary between the different components of the connection and for different rivet clamping stresses. For example, around the holes, $R$ varies between 0.05 and 0.20 in the case of a 100 MPa rivet clamping stress and 0.14 and 0.53 in the case of a $200 \mathrm{MPa}$ rivet clamping stress. Similar variations in $R$ were also found at the rivets and angle fillet. Although different stress ratios would result in an upward or downward shift in the S$\mathrm{N}$ curve, here, the S-N curve shown in Fig. 6(b) is used irrespective of the applied $R$.

The total fatigue damage at each part of the notch is thus calculated by multiplying the damage values obtained from the single train passages (Eq. (3)) by their corresponding annual frequencies given in BS 5400 [4] and summing up over the years for which the bridge is in service. For remaining fatigue life calculations, future load evolution is ignored. It is further assumed that the BS 5400 medium traffic is introduced in 1970 and that fatigue damage up to that time can be considered as negligibly small [12]. 


\section{Results and discussion}

\subsection{Convergence study}

A convergence study was carried out in order to examine the effect of mesh refinement on fatigue damage. Three different mesh densities were investigated parts of which are shown in Fig. 7. Differences between mesh 1 and mesh 2 are brought about by increasing the number of elements along the thickness and along the depth of the angle fillet. For mesh 3, the number of elements along the thickness direction and around the fillet radius is increased further, together with the number of elements along the perimeter of the holes. The rivet mesh corresponding to mesh 1 and mesh 2 is the same, whereas, for mesh 3, the number of elements along the rivet shank and around its perimeter is increased.

Fig. 8 presents the fatigue damage calculated for a single train passage (BS 5400 train No 7) for the three different mesh densities shown in Fig. 7. The results, which correspond to a rivet clamping stress of $100 \mathrm{MPa}$, are presented for a number of locations within the different components of the connection. The results are, at each location, related to the most damaging notch tips.

Comparison of the fatigue damage associated with meshes 1 and 2 reveals considerable differences, which, in some cases, may be as high as 50\%. By increasing the mesh density further (mesh 3), the differences in damage are significantly reduced with a maximum difference of $15 \%$ between meshes 2 and 3. This contrasts the use of the notch tip characteristic stress, which, as is well known, does not converge leading to unbounded fatigue damage as the mesh becomes finer. Fig. 8 suggests that the volume method can alleviate this problem.

The mesh densities used for the convergence study were chosen by considering the practical rules proposed by Chaves and Taylor [28]. In [28], it was observed that in situations where notches having finite root radius $(r>0)$ are replaced by sharp notches (having notch root radius equal to zero) when the ratio $r / L$ is lower than 5 , the zero radius models are conservative and the error is less than $30 \%$. By contrast, zero radius models become too conservative. Regarding mesh density, Chaves and Taylor observed that predictions are accurate and conservative when the ratio between mesh size and $r$ as well as the ratio between mesh size and $L$ are lower than unity. In all other cases, predictions become nonconservative and inaccurate. For all three mesh densities shown in Fig. 7, the above mentioned rules were satisfied in the majority of the cases with only a few exceptions where the ratios were slightly 
above the limits. Notwithstanding these observations, the results presented in Fig. 8 demonstrate that convergence is achieved with increased mesh density.

\subsection{Sensitivity to the critical distance $L$}

In Section 4.1, fatigue damage was found to be relatively mesh-insensitive. However, this damage will, in general, depend on the extent of the volume and hence on the critical distance $L$. In order to investigate the effect of $L$, its value is here varied in a systematic way by considering randomness in fatigue properties. As a starting point, use of Eq. (1) and the equation of the S-N curve leads to

$$
\ln L=\ln \left(\frac{1}{\pi}\right)+2\left\{\ln \left(\Delta \mathrm{K}_{t h}\right)-\frac{1}{m}\left[\ln (C)-\ln \left(N_{F L}\right)\right]\right\}
$$

where $C$ is the constant in the S-N curve. Table 1 shows the parameters appearing in Eq. (4) and the values used in this study. Also shown in Table 1 are the references on which these particular choices were based. In cases where a single value is reported in the literature, this is also assumed to be the mean value. It is worth noting that although, for the threshold parameter, a coefficient of variation $(\mathrm{CoV})$ of 0.1 has been suggested [29], this is here increased to 0.2 due to the larger scatter associated with wrought iron manufacturing.

With the choice of distributions presented in Table 1 and in light of Eq. (4), it follows that $L$ will be lognormally distributed. Although, in general, the threshold stress intensity factor $\Delta K_{t h}$, the fatigue limit $\Delta \sigma_{0}$ and the critical distance $L$ are interrelated with each other, here all the random variables used in Eq. (4) are assumed to be statistically independent. Accordingly, the sensitivity study is carried out on the basis of mean, mean plus one standard deviation and mean minus one standard deviation of this lognormal random variable. The different volumes associated with each value of $L$ are considered for the finest mesh (mesh 3), which was presented in Section 4.1. Moreover, the fatigue damage is calculated based on the mean S-N curve and the passage of the BS 5400 train No 7 [4]. The results, which pertain to a $100 \mathrm{MPa}$ rivet clamping stress are shown in Fig. 9. As can be seen, the estimated fatigue damage displays a degree of sensitivity to the critical distance. This sensitivity appears to depend on the severity of the notch. Thus, for holes associated with the largest notch radius, a small (mean minus one standard deviation) $L$ (and hence volume) appears to result in a considerably higher fatigue damage estimate. In fact, the mean minus one standard deviation fatigue damage estimates, 
relatively to their counterparts associated with the other critical distance measures (mean and mean plus one standard deviation), appear to increase as the notch becomes milder. Moreover, Fig. 9 demonstrates a spatial variability in fatigue damage, which can help explain the fact that in actual bridges different connection components may fail (broken rivets, cracked fillets etc). For example, according to Fig. 9, an $L$ realisation of a mean plus one standard deviation in hole 5, coupled with an $L$ realisation of a mean minus one standard deviation in hole 4, would imply that damage could initiate in hole 4 instead of hole 5 .

Following the sensitivity study on $L$, damage calculations for different BS 5400 trains are presented next using the mean value for $L$ in conjunction with the mean S-N curve. Furthermore, in view of the convergence study presented in Section 4.1, and due to the substantially greater CPU running time associated with the finest mesh, these analyses are carried out using the second mesh density (mesh 2, see Fig. 7).

\subsection{Fatigue damage}

The damage $D_{l l}$ is calculated at each location using the TCD and Eq. (3). As in the case of the convergence study, several notch tips are identified within the location and the results for the most damaged are presented here. Further, the results are compared with those presented in [12]. Therein, in order to obtain the damage $D_{g l}$, calculations were carried out using a nominal stress history obtained from a crude FE model and was coupled with the class pertaining to riveted wrought iron proposed by Network Rail [32]. It should be noted that, in this case, a single fatigue damage estimate is obtained for the entire connection, since damage calculations are carried out with the use of a detail-specific S-N curve.

Fig. 10 depicts the ratio $D_{l l} / D_{g l}$ pertaining to a single BS 5400 medium traffic train, with $D_{l l}$ obtained for a rivet clamping stress of $100 \mathrm{MPa}$. Fig. 11 depicts the same results for a $200 \mathrm{MPa}$ clamping stress. It can be seen in Fig. 10 and 11 that, in most cases, calculations based on the TCD result in lower fatigue damage estimates $\left(D_{l l}<D_{g l}\right)$. However, more crucially, for the critical regions of the connection (hole 5 , rivet 3 and angle fillet), the refined model coupled with the TCD predicts higher fatigue damage for the majority of the trains. Comparison of Fig. 10 and 11 reveals that, for the critical regions, the differences between the predictions of the two models are lower in the case of the higher rivet clamping stress of 200 MPa (Fig. 11). The large differences observed in Fig. 10 and 11 
may be partly attributed to the two-slope S-N curve used in the case of the crude model as opposed to the single slope curve used with the present (TCD) model.

Further damage calculations for an assumed period of 37 years (i.e. from 1970 to present day) are carried out using the stress histories of all four trains comprising the BS 5400 medium traffic. Fatigue damage estimates are obtained using the refined model coupled with the TCD $\left(D_{l}\right)$ and the crude model presented in [12] $\left(D_{g}\right)$. The results are presented in Fig. 12 (clamping stress of $100 \mathrm{MPa}$ ) and Fig. 13 (clamping stress of $200 \mathrm{MPa}$ ), in terms of the ratio $D_{l} / D_{g}$ together with remaining fatigue lives assuming no load evolution.

As in the case of the single trains presented in Fig. 10 and 11, the use of the TCD results in higher damage estimates for the most critical regions of the connection (hole 5, rivet 3 and angle fillet). Depending on the clamping stress, damage can be higher by a factor of 3.5. In these critical regions, the differences with the approach described in [12] are, again, reduced for higher clamping stresses. Comparison of Fig. 12 and 13 reveals the expected beneficial effect of the clamping stress on remaining fatigue life. Fig. 12 and 13 also indicate that hole 5 on the stringer part of the connection (see Fig. 2(b)) is the most highly damaged part irrespective of the level of clamping. As a result of outof-plane deformations, rivet 3 on the cross-girder part of the connection (see Fig. 2(b)) appears to be the next most highly damaged part for $100 \mathrm{MPa}$ rivet clamping stress (see Fig. 12), whereas the angle fillet assumes second place for a 200 MPa clamping stress.

It is worth noting that damage ranking of the different connection components estimated by using the TCD (Fig. 10 to 13) is different from the ranking obtained in [6] where notch stresses were considered. As mentioned previously, points of maximum stress concentration may not be necessarily associated with the highest fatigue damage, which according to the TCD is influenced by the stress distribution ahead of the notch. The results presented in Fig. 10-13 should be viewed within the uncertainty context associated with manufacturing (clamping stress) and material properties (critical distance, S-N curves).

The results presented in this paper were obtained by assuming that in the finite life region the critical distance can be kept constant and equal to its value determined in a near threshold condition. However, recently it has been shown by Susmel and Taylor [33] that, in order to coherently extend the use of the TCD down to the medium-cycle fatigue regime, the critical distance value has to increase as the number of cycles to failures decreases. The assumption used in this paper, i.e. to keep the critical 
distance value constant, seems reasonable in practice because it allows a higher margin of safety to be reached and it will always result in conservative fatigue life estimates when the TCD is used to estimate fatigue damage in the medium-cycle fatigue regime. The fact that the critical distance value in the low/medium-cycle fatigue regime is approximately 10 times higher than the corresponding highcycle fatigue value may explain better the high degree of conservatism shown by the analyses presented in this paper.

\section{Conclusions}

Following the work carried out in [6], this paper has presented fatigue damage estimates for a typical, short-span, riveted railway bridge. Calculations were performed using the Theory of Critical Distances (TCD). The TCD, rather than relying on a single, remotely applied stress or the stress at the notch, considers the entire stress field ahead of the notch. This study has demonstrated that, with FE mesh refinement, reasonable convergence is achieved through the use of the TCD. This contrasts the case of considering notch stresses where fatigue damage, with increasing mesh density, becomes unbounded.

By considering the random characteristics of the critical distance $L$, fatigue damage under the passage of a single train was found to be relatively sensitive to the choice of $L$. Following this sensitivity study, damage estimates obtained using the TCD were compared with the estimates calculated using the traditional approach i.e., a remotely applied stress coupled with the detail-specific S-N curve. Calculations were carried out for different trains and the differences between the two methods were on the whole found to decrease for larger values of the rivet clamping stress. Under a combined load history, the traditional approach tends to underestimate the fatigue damage of the critical regions of the connection, by a maximum factor of 3.5 in the case of low rivet clamping force.

The appealing features of the TCD are its reliance on straightforward material characterisation and its convergent characteristics. On the other hand, the method requires, at least for 3D bridge applications, considerable post-processing and is in need of further validation using appropriate test results. 


\section{References}

[1] Bellett D, Taylor D, Marco S, Mazzeo E , Guillois J, Pircher T. The fatigue behaviour of threedimensional stress concentrations. Int J Fatigue 2005; 27(3):207-21.

[2] Al-Emrani M. Fatigue in riveted railway bridges - A study of the fatigue performance of riveted stringers and stringer-to-floor-beam connections. PhD Thesis. Chalmers University of Technology, Sweden, 2002.

[3] Imam B. Fatigue analysis of riveted railway bridges. PhD Thesis. University of Surrey, UK, 2006.

[4] BS 5400. Steel, concrete and composite bridges: Part 10: Code of practice for fatigue. British Standards Institute, London, 1980.

[5] American Association of State Highway Transportation Officials (AASHTO). LRFD bridge design specifications. $2^{\text {nd }}$ ed., Washington, D. C., 1998.

[6] Imam BM, Righiniotis TD, Chryssanthopoulos MK. Numerical modelling of riveted railway bridge connections for fatigue evaluation. Engineering Structures 2007; 29(11):3071-81.

[7] Dong P. A structural stress definition and numerical implementation for fatigue analysis of welded joints. Int J Fatigue 2001;23(10):865-76.

[8] Susmel L. The theory of critical distances: a review of its applications in fatigue. Eng Fract Mech 2007; doi:10.1016/j.engfracmech.2006.12.004.

[9] DNV. Fatigue design of offshore steel structures. Recommended Practice, DNV-RP-C203. Det Norske Veritas, Norway, 2005.

[10] Doerk O, Fricke W, Weissenborn C. Comparison of different calculation methods for structural stresses at welded joints. Int J Fatigue 2003;25(5):359-69.

[11] Taylor D. Geometrical effects in fatigue: a unifying theoretical model. Int J Fatigue 1999;21(5):413-20.

[12] Imam B, Righiniotis TD, Chryssanthopoulos MK, Bell B. Analytical fatigue assessment of a typical riveted UK rail bridge. Proceedings of the Institution of Civil Engineers (ICE) - Bridge Engineering 2006;159(3):105-16.

[13] Neuber H. Theory of notch stresses: principles for exact calculation of strength with reference to structural form and material. Berlin: Springer, 1958. 
[14] Peterson RE. Notch sensitivity. In: Sines G, Waisman JL, editors. Metal fatigue. New York: McGraw Hill, 1959:293-306.

[15] Taylor D, Wang G. The validation of some methods of notch fatigue analysis. Fatigue Fract Engng Mater Struct 2000;23(5):387-94..

[16] Taylor D, Bologna P, Bel Knani K. Prediction of fatigue failure location on a component using a critical distance method. Int J Fatigue 2000;22(9):735-42.

[17] Taylor D. Analysis of fatigue failures in components using the theory of critical distances. Engng Fail Anal 2005;12(6):906-14.

[18] Susmel L, Taylor D. Fatigue design in the presence of stress concentrations. J Strain Anal Eng Design 2003;38(5):443-52.

[19] Taylor D, Barrett N, Lucano G. Some new methods for predicting fatigue in welded joints. Int J Fatigue 2002;24(5):509-18.

[20] Crupi G, Crupi V,Guglielmino E, Taylor D. Fatigue assessment of welded joints using critical distance and other methods. Engng Fail Anal 2005;12(1):129-42.

[21] Susmel L, Taylor D. A simplified approach to apply the theory of critical distances to notched components under torsional fatigue loading. Int J Fatigue 2006;28(4):417-30.

[22] Susmel L. A unifying approach to estimate the high-cycle fatigue strength of notched components subjected to both uniaxial and multiaxial cyclic loadings. Fatigue Fract Engng Mater Struct 2004;27(5):391-411.

[23] Araujo JA, Susmel L, Taylor D, Ferro JCT, Mamiya EN. On the use of the theory of critical distances and the modified Wohler curve method to estimate fretting fatigue strength of cylindrical contacts. Int J Fatigue 2007;29(1):95-107.

[24] Cullimore MSG. The fatigue strength of wrought iron after weathering in service. The Structural Engineer 1967;45(5):193-9.

[25] Brühwiler E, Smith IFC, Hirt MA. Fatigue and fracture of riveted bridge members. J Str Eng 1990;116(1):198-214.

[26] Moy SSJ, Clark J, Clarke H. The strengthening of wrought iron using carbon fibre reinforced polymer composites. In: Proc Advanced Polymer Composites for Structural Applications in Construction (ACIC 2004). University of Surrey, UK, 2004:258-65. 
[27] Helmerich R, Kühn B, Nussbaumer A. Assessment of existing steel structures. A guideline for estimation of the remaining fatigue life. Structure and Infrastructure Eng 2007;3(3):245-55.

[28] Chaves V, Taylor D. Use of simplified models in fatigue prediction of real components. In: Proc $8^{\text {th }}$ Int Fatigue Conf., Fatigue 2002, Vol. 5, Stockholm, 2002: 2799-2806.

[29] Taylor D. Fatigue thresholds. London: Butterworths, 1989.

[30] Joint Committee on Structural Safety. The probabilistic model code. Internet Publication. http://www.jcss.ethz.ch, 2001.

[31] Gurney, TR. The basis of the new fatigue design rules for welded joints. In Rockey KC and Evans HR, editors. The Design of Steel Bridges. London: Granada, 1982:475-96.

[32] Railtrack. RT/CE/C/025, Railtrack line code of practice: The structural assessment of underbridges, Railtrack, 2001.

[33] Susmel L, Taylor D. A novel formulation of the Theory of Critical Distances to estimate lifetime of notched components in the medium-cycle fatigue regime. Fatigue Fract Engng Mater Struct 2007;30(7):567-581. 

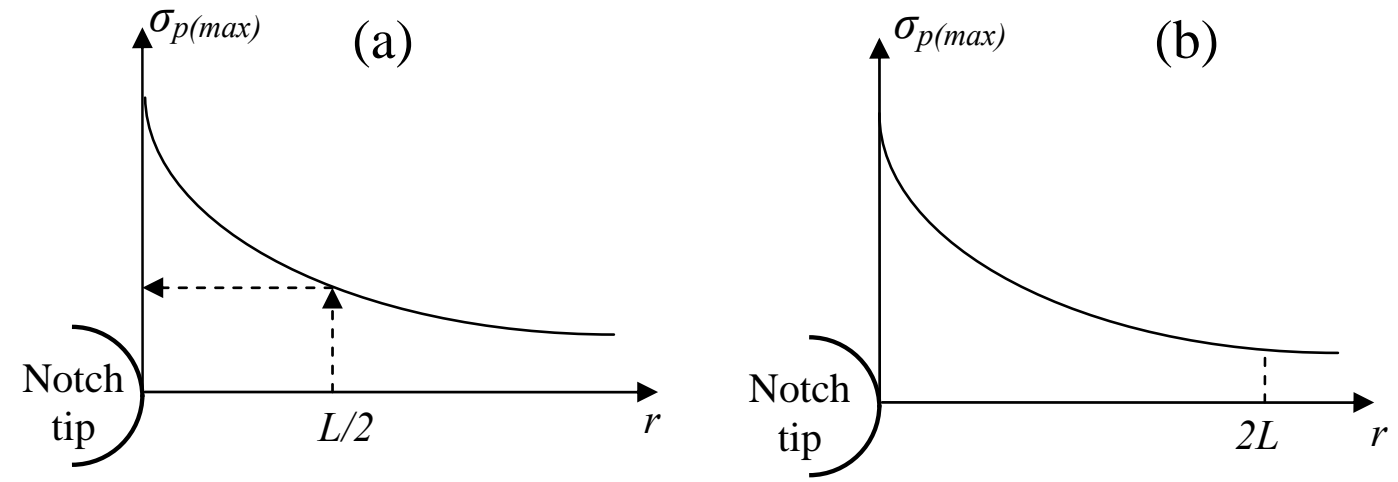

Fig. 1. Illustration of the (a) point method and (b) line method. 


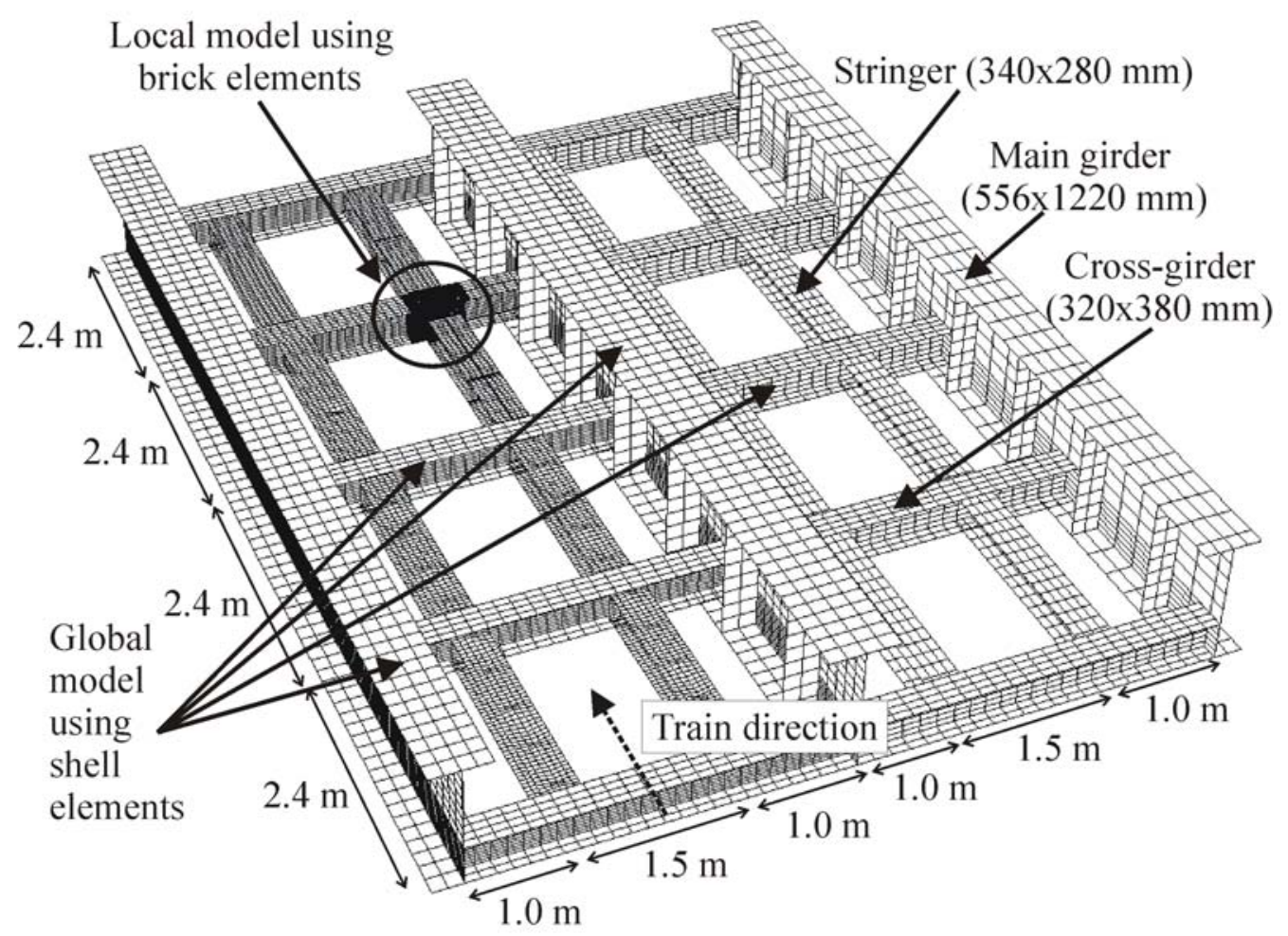

(a)

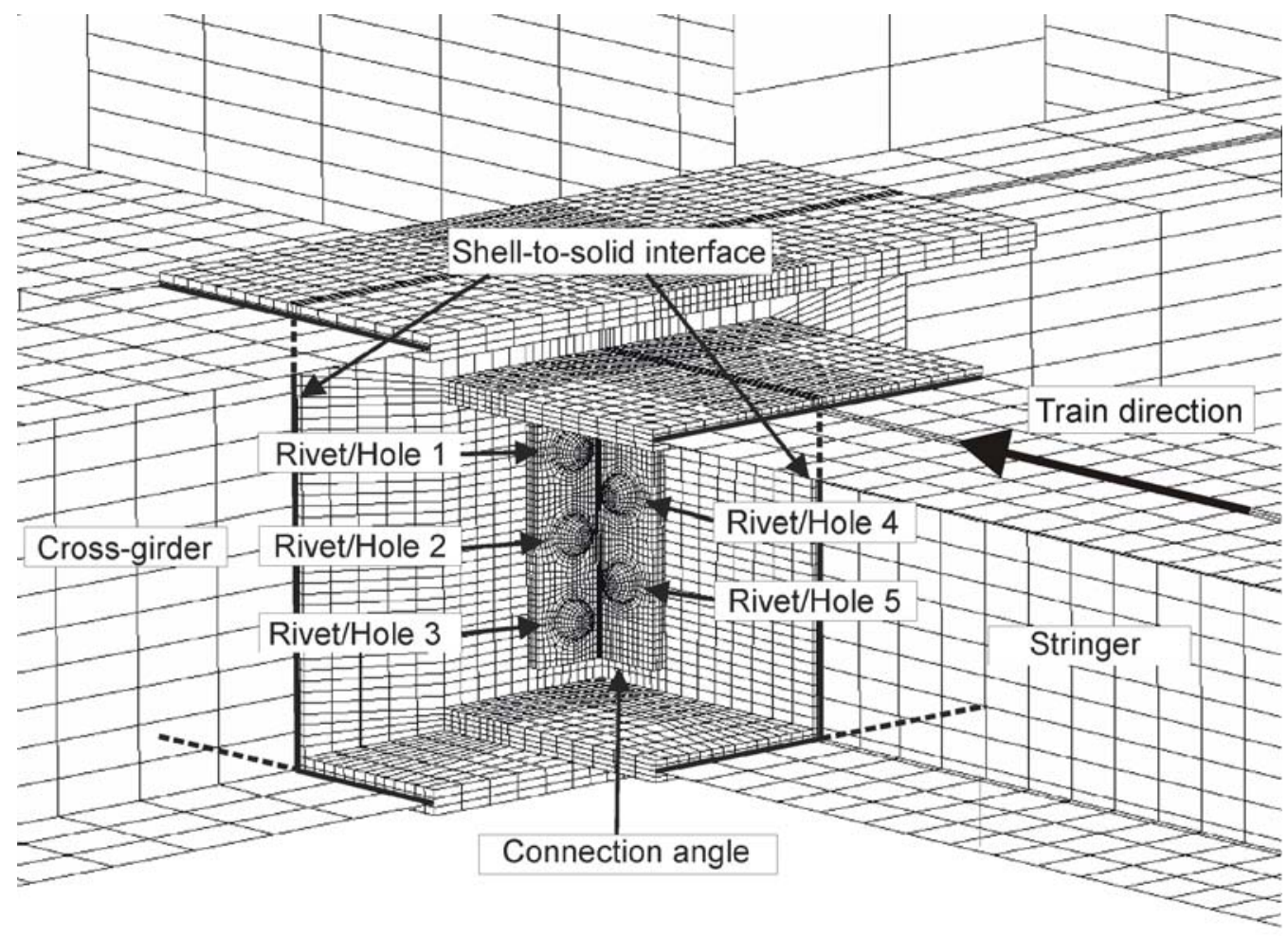

(b)

Fig. 2. (a) Global-local finite element model of the riveted bridge and (b) Close-up view of the connection and hole/rivet nomenclature. 


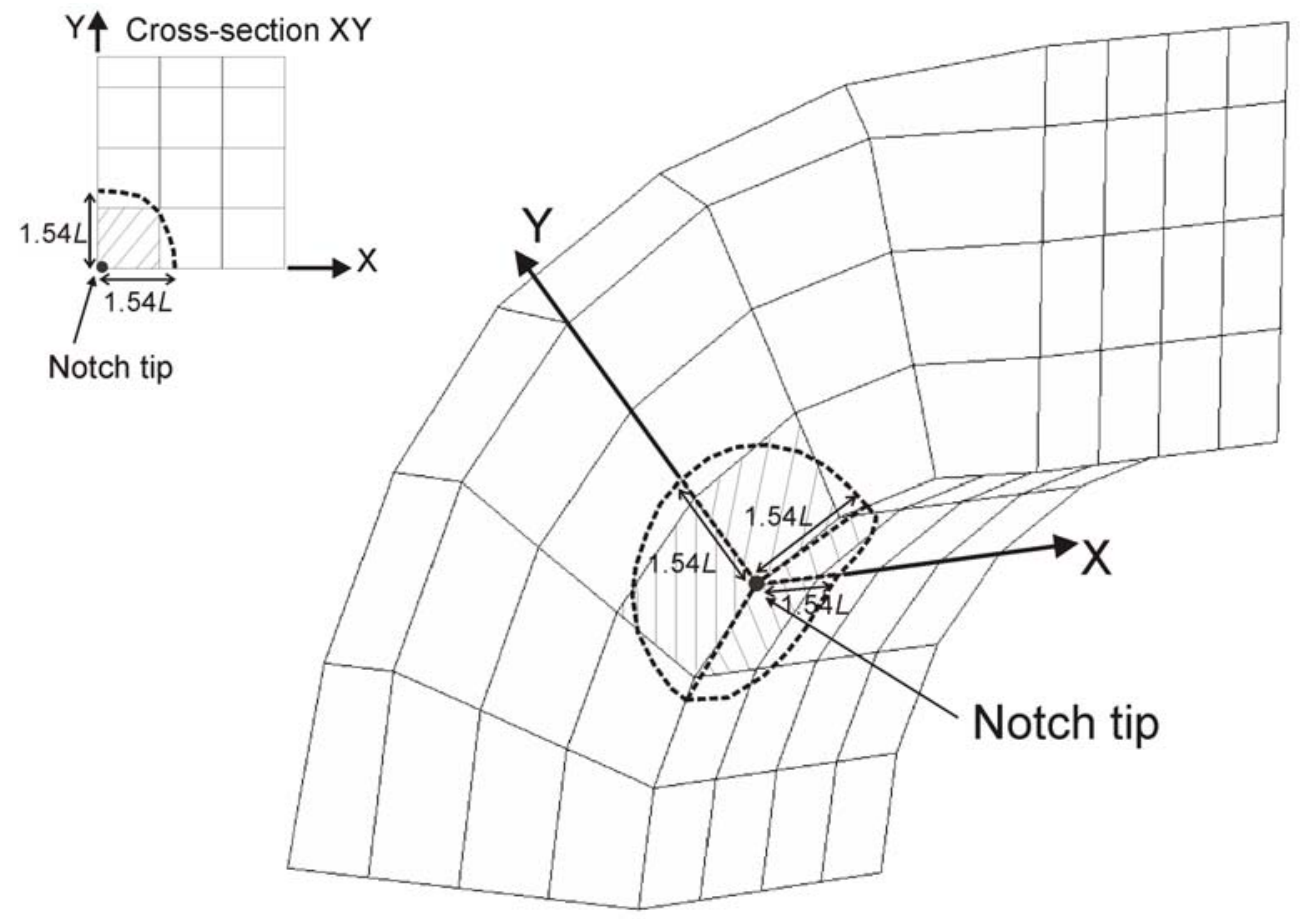

Fig. 3. Critical volume around the hole perimeter. 


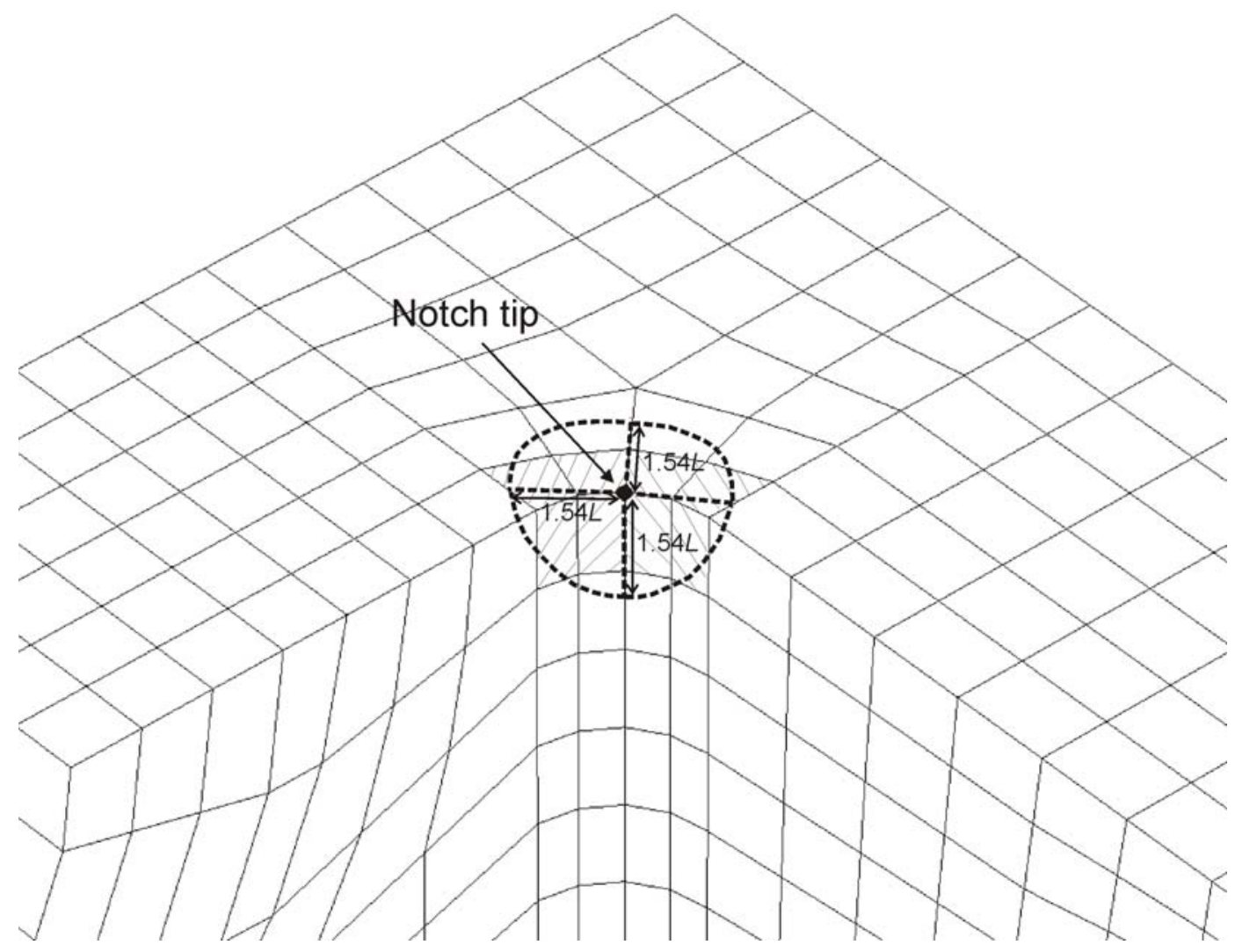

Fig. 4. Critical volume around the angle fillet. 

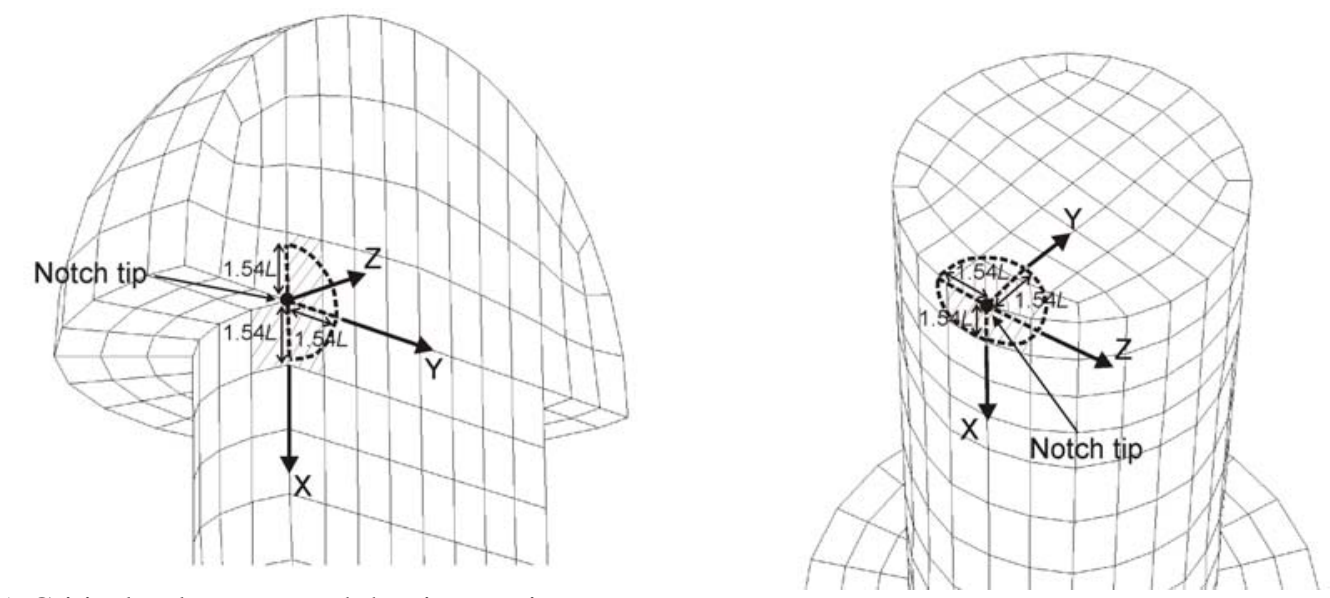

Fig. 5. Critical volume around the rivet perimeter. 


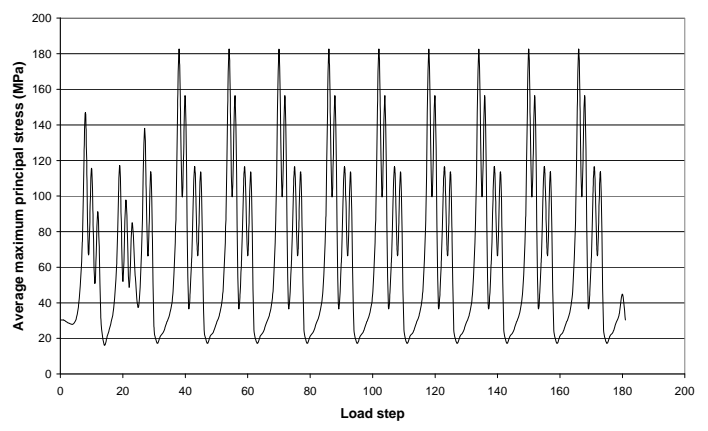

(a)

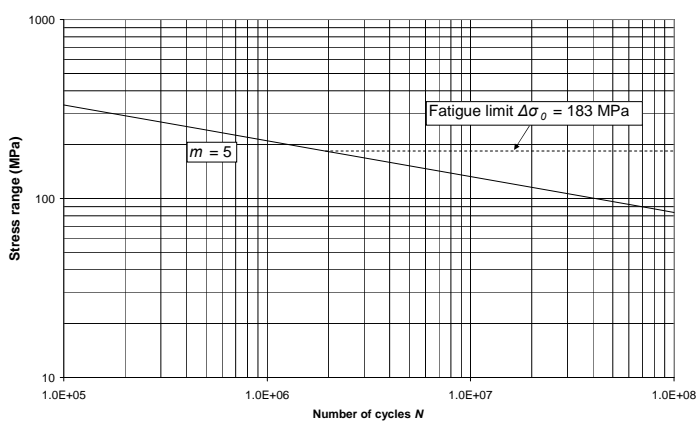

(b)

Fig. 6. (a) Stress history at edge of hole 5; (b) S-N curve for plain wrought iron [24]. 

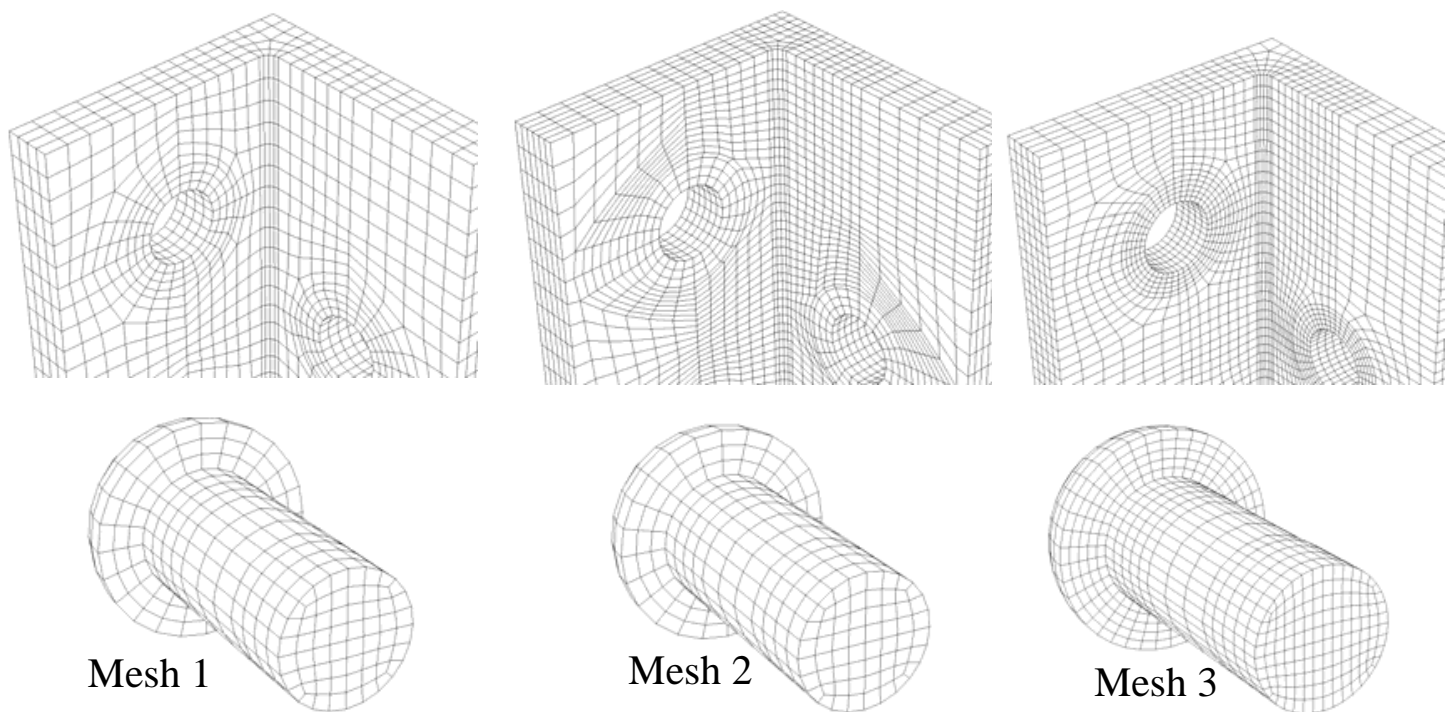

Fig. 7. Parts of the FE model pertaining to three different mesh densities. 


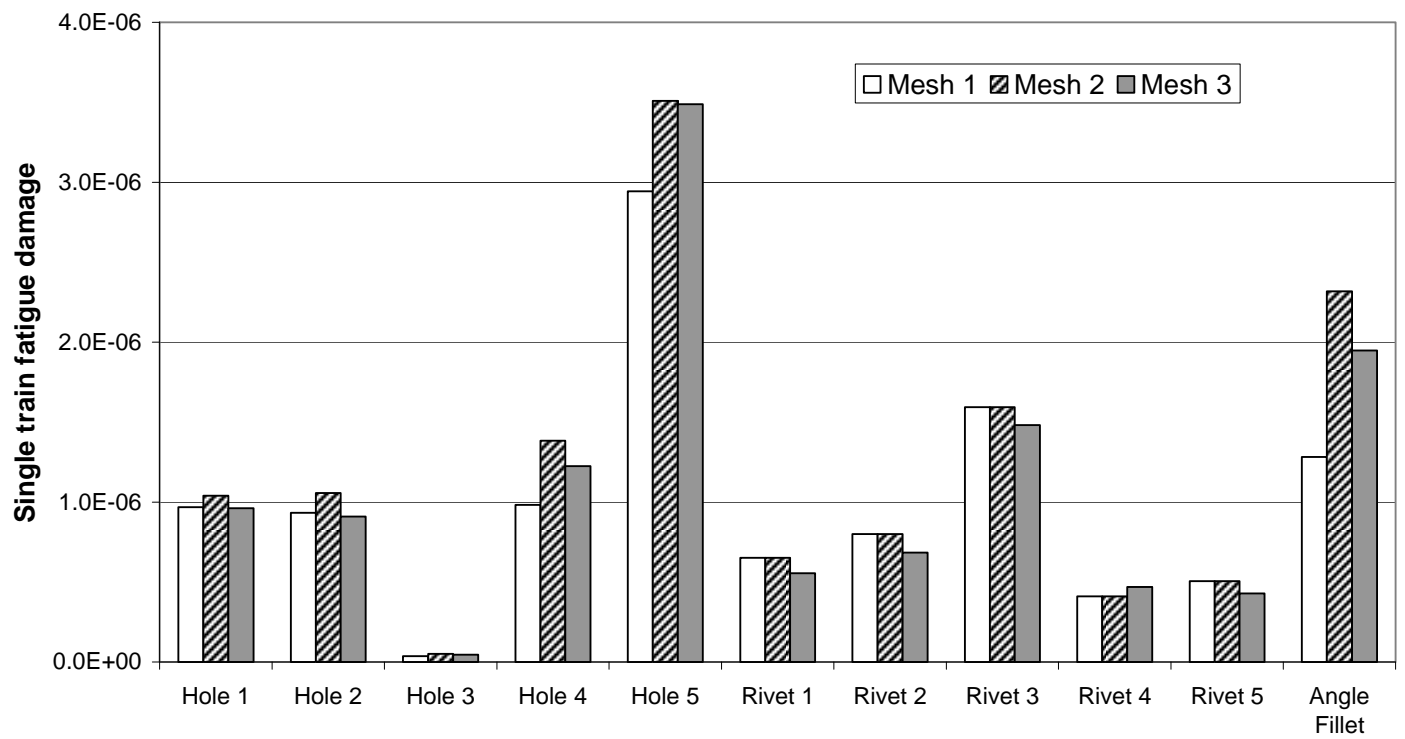

Fig. 8. Fatigue damage at different locations of the connection for three different mesh densities (BS 5400 train No 7, rivet clamping stress $=100 \mathrm{MPa}$ ). 


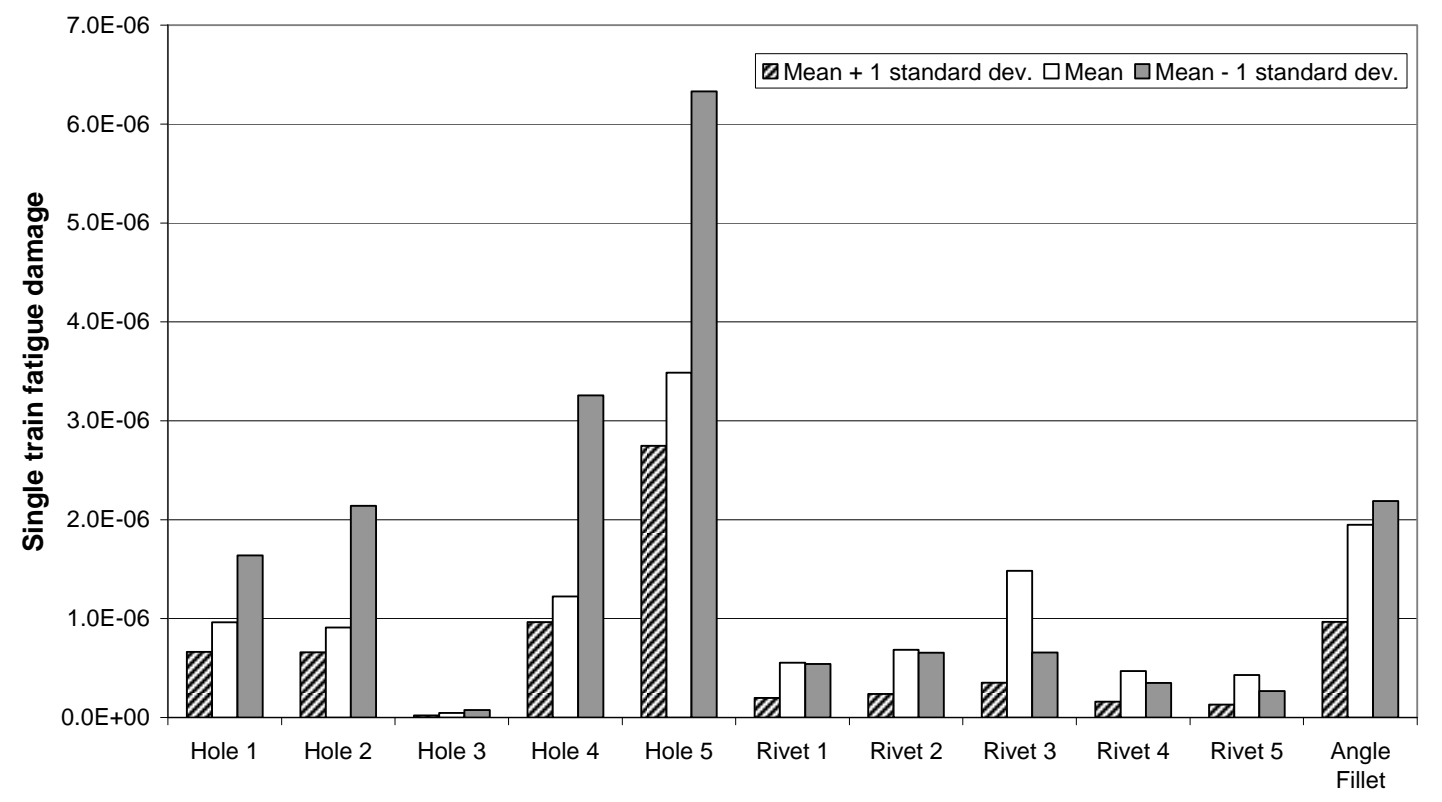

Fig. 9. Fatigue damage at different locations of the connection for three different critical distance values (BS 5400 train No 7, rivet clamping stress $=100 \mathrm{MPa}$ ). 


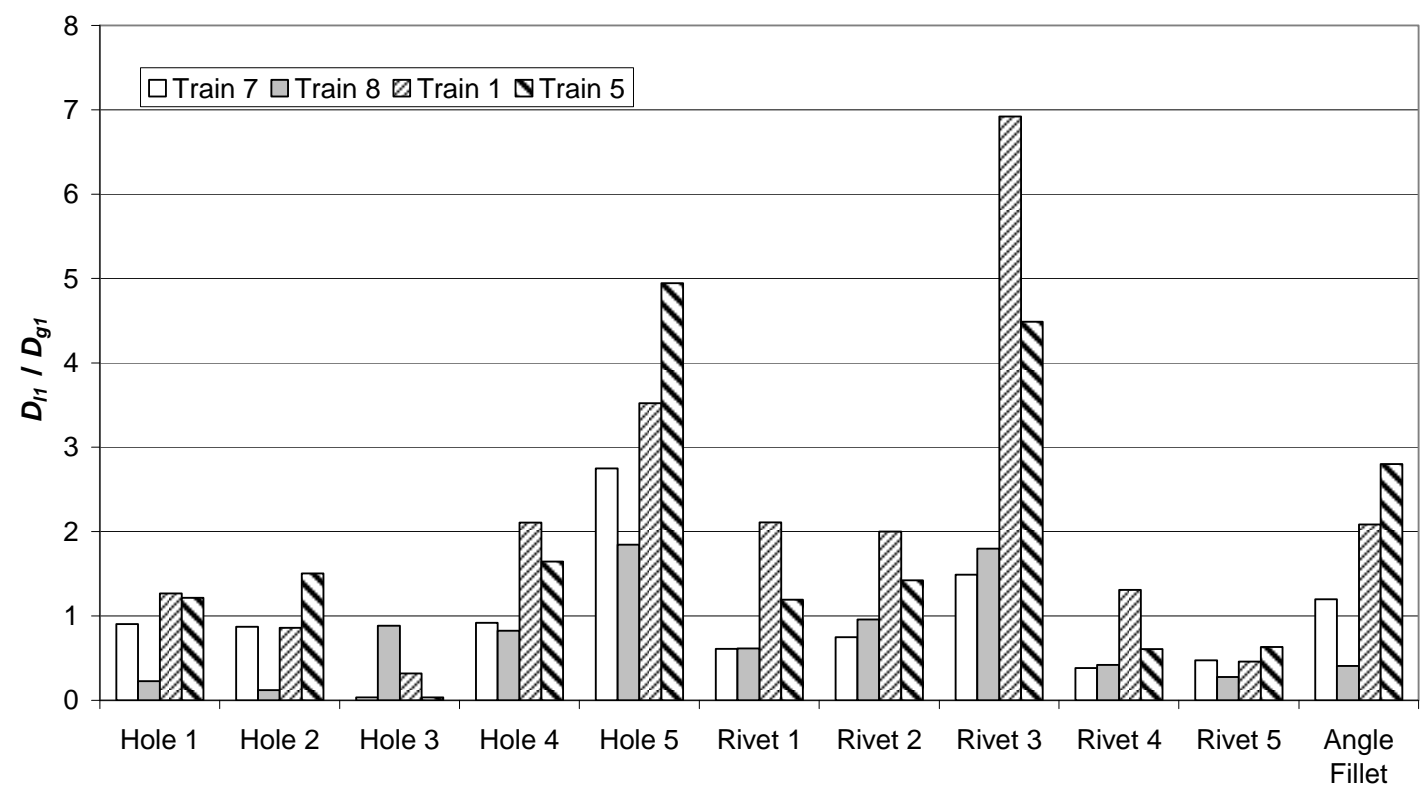

Fig. 10. Comparison of fatigue damage between crude [12] and refined (TCD) models, for each of the BS 5400 medium traffic trains (single train passage; rivet clamping stress = $100 \mathrm{MPa}$ ). 


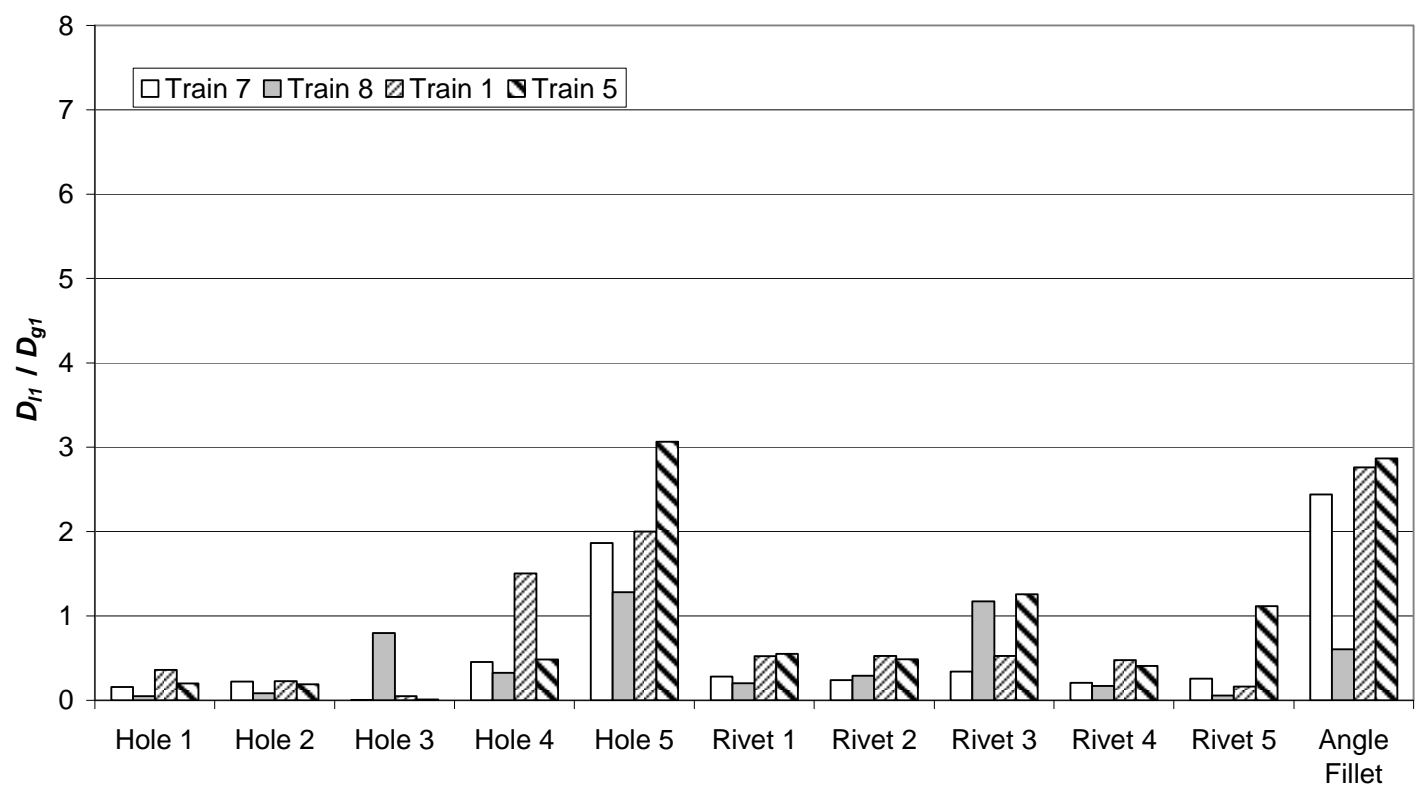

Fig. 11. Comparison of fatigue damage between crude [12] and refined (TCD) models, for each of the BS 5400 medium traffic trains (single train passage; rivet clamping stress = $200 \mathrm{MPa}$ ). 


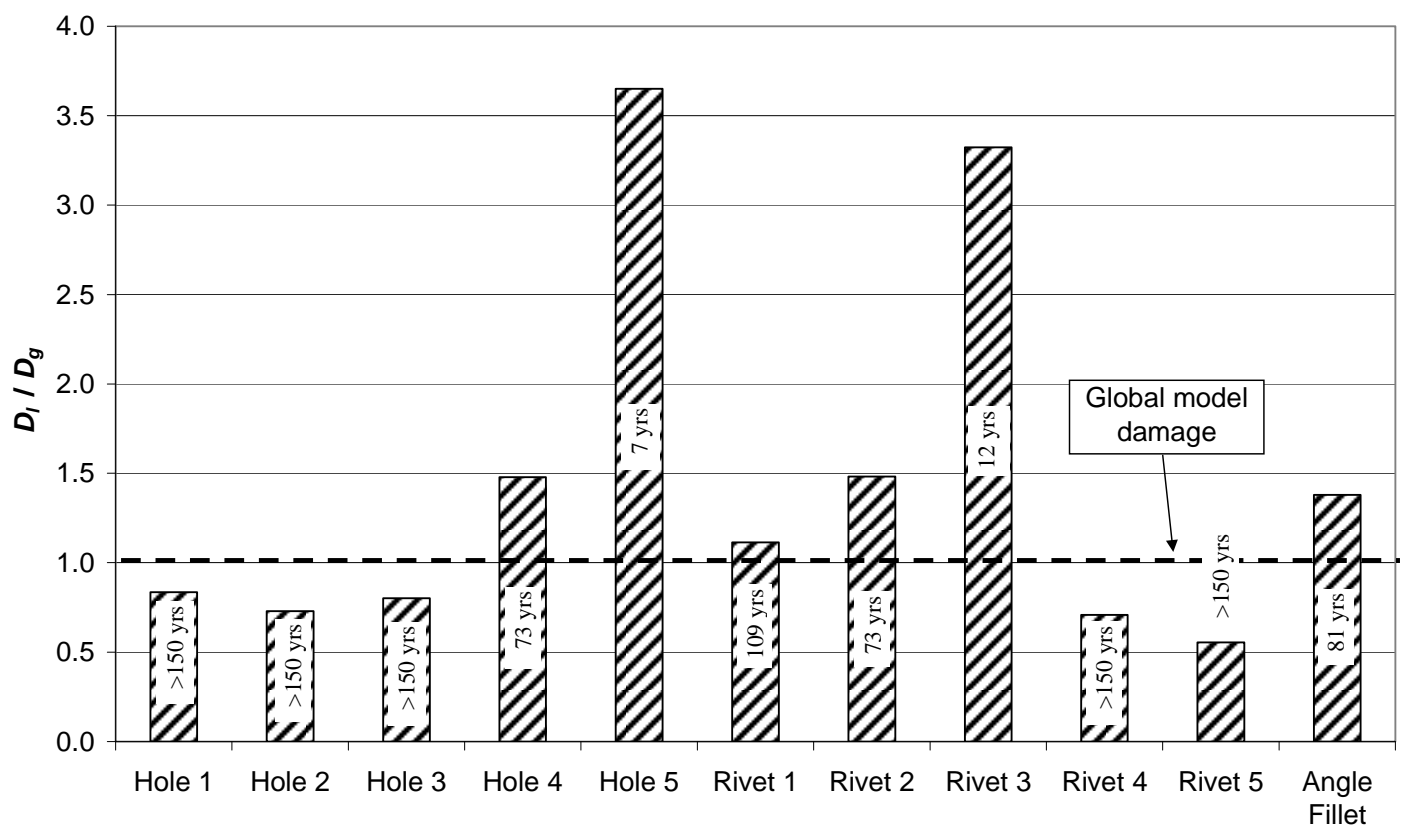

Fig. 12. Comparison of total fatigue damage between crude [12] and refined (TCD) models for the period 1970-2007 and corresponding remaining fatigue lives (BS 5400 medium traffic; rivet clamping stress $=100 \mathrm{MPa})$. 


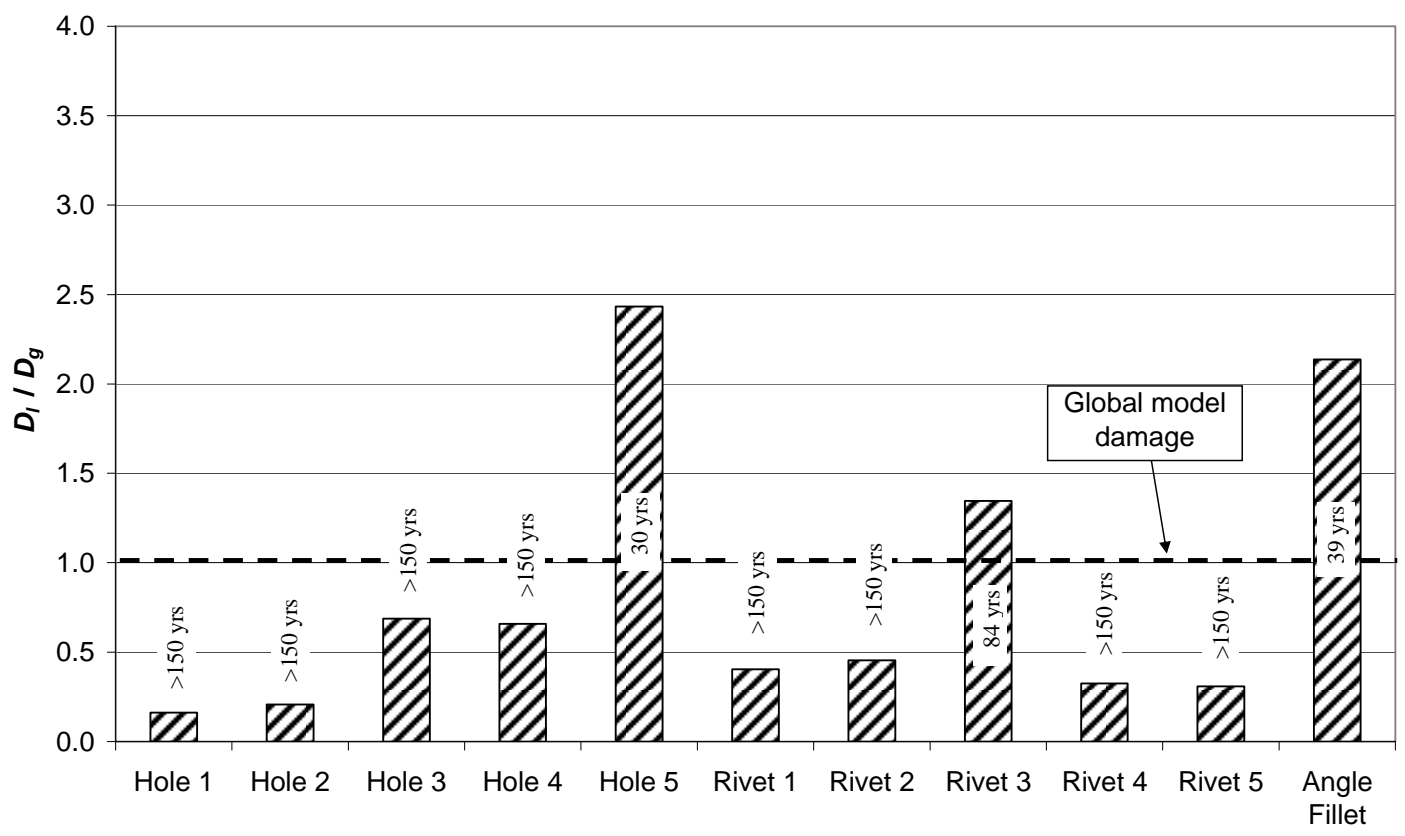

Fig. 13. Comparison of total fatigue damage between crude [12] and refined (TCD) models for the period 1970-2007 and corresponding remaining fatigue lives (BS 5400 medium traffic; rivet clamping stress $=200 \mathrm{MPa}$ ). 
Table 1. Parameters used in Eq. (4)

\begin{tabular}{cccc}
\hline Variable & Mean & CoV & Distribution Type \\
\hline$\Delta K_{t h}$ & $13.5 \mathrm{MPa} \cdot \mathrm{m}^{1 / 2}[27]$ & 0.2 & Lognormal [29] \\
$C$ & $4.1 \times 10^{17}[24]$ & $0.3[4]$ & Lognormal [30] \\
$m$ & $5[24]$ & 0 & Deterministic [29] \\
$N_{F L}$ & $2 \times 10^{6}[24]$ & 0 & Deterministic \\
\hline
\end{tabular}

